\title{
Insight into the management of non-traumatic perforation of the small intestine
}

\author{
Bhupendra Kumar Jain, Himanshu Arora, Upendra Kumar Srivastava, Debajyoti Mohanty, \\ Pankaj Kumar Garg
}

Department of Surgery, University College of Medical Sciences and Guru Teg Bahadur Hospital, University of Delhi, Delhi, India

\begin{abstract}
Introduction: Management of non-traumatic perforation of the small intestine has always been a consideration for surgeons because of associated enormous morbidity and mortality. There is a paucity of data on the management of non-traumatic perforation of the small intestine.

Methodology: A retrospective study was conducted which involved analysis of 192 patients treated for non-traumatic perforation of small intestine in a tertiary care teaching hospital in North India. The clinical profile and management of the patients were studied.

Results: The most common cause of non-traumatic perforation of small intestine was typhoid (46.4\%), followed by non-specific inflammation $(39.2 \%)$, tuberculosis $(12.8 \%)$ and malignant neoplasm $(1.6 \%)$. Primary repair was the most frequent procedure $(44.0 \%)$, followed by ileostomy $(25.5 \%)$ and resection-anastomosis $(19.3 \%)$. Superficial wound infection was the most frequent postoperative complication $(46.8 \%)$, followed by wound dehiscence $(31.3 \%)$. The wound infection rate was reduced significantly following delayed primary closure of skin incision. Enterocutaneous fistula/leak developed in $11.5 \%$ patients. Salvage ileostomy for post-operative intestinal leak resulted in a better survival rate as compared to conservative treatment ( $85.7 \%$ vs. $50.0 \%)$. The overall mortality rate was $16.6 \%$.

Conclusion: Operative procedures undertaken for the management of non-traumatic perforation of small intestine can be classified into two groups: procedures that leave an intestinal suture line inside the peritoneal cavity and procedures that do not. The no suture line-in procedure seems to be better option in adverse patient conditions.
\end{abstract}

Key words: Perforation peritonitis; operative strategies; complications

J Infect Dev Ctries 2010; 4(10):650-654.

(Received 13 January 2010 - Accepted 25 April 2010)

Copyright (C) 2010 Jain et al. This is an open-access article distributed under the Creative Commons Attribution License, which permits unrestricted use, distribution, and reproduction in any medium, provided the original work is properly cited.

\section{Introduction}

Non-traumatic perforation of the small intestine is one of the common surgical emergencies encountered by surgeons in developing countries. Causes of non-traumatic perforation of the small intestine in developing countries are clearly different from those observed in developed countries [1]. Patients often present late with purulent peritonitis and poor general condition [2]. As a result, serious complications such as post-operative peritonitis caused by a leak from repaired intestine, superficial wound infection, and complete wound dehiscence are not uncommon. The management of complications is particularly difficult in developing countries due to limited resources, particularly facilities for parenteral nutrition. The present retrospective study was undertaken to evaluate the existing management practices and outcomes in patients operated for non- traumatic perforation of the small intestine at our institution.

\section{Methodology}

A retrospective study was conducted in the Department of Surgery at University College of Medical Sciences and Guru Teg Bahadur Hospital, Delhi.

\section{Data collection}

A total of 192 patients of non-traumatic perforations of the small intestine operated in emergency surgery over a period of two years were analyzed. The patients with intestinal perforations secondary to mesenteric ischemia and obstruction or strangulation due to intra-abdominal adhesions, hernia, or volvulus were excluded from the study. 


\section{Patients and management}

All the patients admitted with a tentative diagnosis of non-traumatic perforation of the small intestine were clinically assessed and adequately resuscitated with administration of intravenous fluids, antibiotics, and placement of nasogastric tube. A close watch was kept on the vital signs and urine output. Appropriate measures were taken to correct hemoglobin, blood sugar, blood urea, and serum electrolytes expeditiously. Plain abdominal X-rays were performed for evidence of pneumo-peritoneum. Contrast enhanced CT scan (CECT) of the abdomen could not be undertaken in these patients due to unavailability. A decision of laparotomy was made on clinical grounds supplemented by investigations.

At laparotomy, the operative findings were noted and the volume and nature of the exudates/ intestinal contents drained from the peritoneal cavity were recorded. The intestinal perforation was managed by one or more of the following procedures:

1. The edge of the perforation was excised and closed transversely in one layer by using number 2-0 silk/polyglactin.

2. Resection of the unhealthy segment of the intestine was done followed by end-to-end anastomosis (Ileo-ileal, end-to-side ileotransverse, or side-to-side ileo-transverse).

3. An ileostomy was made either by exteriorizing the bowel at the site or proximal to perforation (loop diverting ileostomy) or by exteriorizing end(s) of the ileum after resection of the unhealthy segment containing the perforation.

The peritoneal cavity was thoroughly lavaged with normal saline. Tube drains were placed to drain the pelvis and the paracolic gutters. The rectus sheath was closed by continuous no. 1 non-absorbable suture. The skin was approximated with either sutures or staples. In patients with gross peritoneal contamination (feculent and of a volume of more than one liter), the skin incision was not primarily closed on conclusion of laparotomy, but later on the fourth post-operative day as delayed primary closure. All patients received postoperative antibiotic therapy with ceftriaxone + metronidazole \pm amikacin.

Attention was paid to major complications such as pulmonary complications, wound infection, wound dehiscence, residual intra-abdominal abscesses, and enterocutaneous fistula/leak. Appropriate measures, including a second operation, if required, were undertaken to manage the complications.

\section{Statistical analysis}

Analysis was performed using SPSS version 10.0 Statistical packages for windows (SPSS, Chicago, Illinois). Categorical data was compared using Pearson's chi-square test while Fisher's exact test was used to analyze data where cell frequency was small $(<5)$. Continuous data was analyzed by the Mann-Whitney (nonparametric) rank sum test. All $P$ values were considered significant at $<0.05$ (2 tailed).

\section{Results}

The study included 192 patients with a mean age of 29.3 \pm 12.3 years (range 13-78 years) and a male to female ratio of 5:1. Duration of symptoms ranged from one to 12 days with a median of six days. Abdominal pain was the constant symptom present in all patients $(100.0 \% ; \mathrm{n}=192)$, while fever $(70.8 \% ; \mathrm{n}$ $=136)$, obstipation $(55.2 \% ; \mathrm{n}=106)$, and vomiting $(35.9 \% ; \mathrm{n}=69)$ were the other frequent symptoms. Twenty-nine patients $(15.1 \%)$ were operated on within 24 hours of developing perforation (duration of symptoms) and 125 patients $(65.1 \%)$ were operated on between one and four days of developing perforation. The remaining 38 patients (19.7\%) presented to us after four days of developing perforation. Most of the patients $(92.1 \% ; \mathrm{n}=177)$ were operated on within 16 hours of hospital admission.

\section{Operative findings}

There was one perforation in 165 patients (85.9\%) and two perforations in 15 patients (7.8\%). The remaining 12 patients $(6.2 \%)$ had three or more perforations. Ninety percent of the perforations were located in distal $100 \mathrm{~cm}$. of ileum. Peritoneal contamination was assessed in terms of volumes of peritoneal exudates/ intestinal contents evacuated from the peritoneal cavity and its nature (reactionary, purulent and feculent). Contamination was less than one litre in $56.7 \%(\mathrm{n}=109)$ of patients; in the remaining $43.3 \%$ patients $(n=83)$, it was more than one litre.

\section{Operative procedures}

Primary repair of the perforation was the most frequently performed procedure $(44.2 \% ; \mathrm{n}=85)$, followed by ileostomy $(25.5 \%$; $n=49)$, resectionanastomosis $(19.3 \%$; $\mathrm{n}=37)$, and a combination of these procedures in the remaining $10.9 \%(\mathrm{n}=21)$ patients. Skin incision was not closed primarily on conclusion of laparotomy in $19.5 \%(n=37)$ of the 
patients. The data was analyzed to determine a significant association between several preoperative factors and choice of operative procedure. Documentation of shock at presentation, the presence of two or more perforations, and intraoperative contaminant volume of more than one litre appeared to favor the choice of ileostomy over primary closure or resection/anastomosis; however, this association was found to be statistically significant in the case of the last factor only $(53.0 \%$ versus $18.9 \% ; P=0.000$, chi square test).

\section{Postoperative complications}

Superficial wound infection was the most frequent postoperative complication detected in $46.8 \%$ ( $\mathrm{n}=90)$ of patients, followed by wound dehiscence $(31.3 \% ; n=50)$ (Table 1). Occurrence of wound infection had positive correlation with a perforation operation interval of greater than 48 hours (58.2\% versus $34.8 \% ; P=0.004$, chi square test) and the presence of two or more perforations (70.4\% versus $43.0 \% ; P=0.008$, chi square test). It was observed that the wound infection rate was reduced by a factor of 0.4 following delayed primary closure of skin incision as compared to primary closure (22.7\% versus $54.9 \% ; P=0.007$, chi square test).

Post-operative enterocutaneous fistula/leak developed in 22 patients (11.5\%); however, only $2.0 \%$ of these developed following ileostomy while $31.3 \%$ developed when the primary repair was covered with proximal ileostomy. Enterocutaneous fistula/leak occurred in $14.1 \%$ and $10.8 \%$ of patients following simple closure and resection-anastomosis, respectively. These 22 patients were managed by either salvage ileostomy (14 patients) or conservatively (eight patients). Twelve of the fourteen patients $(85.7 \%)$ who underwent salvage ileostomy survived, in comparison to four of eight patients $(50.0 \%)$ who were managed conservatively following intestinal leak.

Thirteen patients developed intra-abdominal abscess and seven of these patients required drainage. One patient required revision of ileostomy for retracted stoma.

\section{Mortality}

The overall mortality was $16.6 \%$ (31 out of 192 patients). The mortality had a positive correlation with the presence of shock at admission (35.3\% versus $14.3 \% ; P=0.036$, chi square test), and peritoneal contaminant fluid volume above one liter ( $29.0 \%$ versus $7.7 \% ; P=0.005$, chi square test). The mortality showed a positive correlation with increasing perforation-to-operation interval. The mortality rate was $3.4 \%$ for 29 patients whose perforation-to-operation interval was less than 24 hours, while it was $39.5 \%$ for 38 patients whose perforation-to-operation interval was greater than 96 hours. The difference was statistically significant $(P$ $=0.000$, chi square test). The mean hospital stay of the patients $(\mathrm{n}=192)$ was $13.7 \pm 9.1$ days (median 11.0 days; range 1-63 days).

\section{Etiology of intestinal perforation}

The most common cause of non-traumatic perforation of the small intestine was typhoid $(46.4 \%)$, followed by non-specific inflammation (39.2\%), tuberculosis (12.8\%) and malignant neoplasm (1.6\%). Non-specific inflammation was diagnosed when biochemical (Widal test negative) and histopathological analysis revealed no specific cause.

Table 1. Postoperative complications in 192 patients of non traumatic perforation of small intestine

\begin{tabular}{|l|l|}
\hline Complications & No. of Patients (\%) \\
\hline Wound infection & $90(46.8)$ \\
\hline Partial wound dehiscence & $38(19.8)$ \\
\hline Complete wound dehiscence & $22(11.5)$ \\
\hline Enterocutaneous fistula/ leak & $22(11.5)$ \\
\hline Intra-abdominal abscesses & $13(06.8)$ \\
\hline Retraction of ileostomy & $01(00.5)$ \\
\hline $\begin{array}{l}\text { Cardiopulmonary } \\
\text { complications }\end{array}$ & $38(19.8)$ \\
\hline Septicemia & $32(16.7)$ \\
\hline Death & $31(16.6)$ \\
\hline
\end{tabular}

\section{Discussion}

Various authors have recommended a variety of operative procedures for treatment of small intestinal perforations: simple closure [3,4]; resection and anastomosis $[5,6]$; closure or resection with bypass procedure [7]; and ileostomy [8-10]. Operative procedures undertaken for the management of nontraumatic perforation of the small intestine can be classified into two groups: (a) procedures which leave an intestinal suture line inside the peritoneal cavity: for example, primary closure of perforation, resection-anastomosis of the affected segment of intestine, or closure of the perforation and bypass; (b) 
procedures which leave no intestinal suture line in the peritoneal cavity: for example, ileostomy. In our study, 49 patients underwent ileostomy which did not leave a suture line in the peritoneal cavity, while 143 patients underwent procedures which left a suture line in the peritoneal cavity. The post-operative intestinal leak rate was only $2.0 \%$ in the former group while it was $14.8 \%$ in the second group. The suture line-in procedures present a considerable risk of intestinal leakage if the suture line does not heal satisfactorily due to the presence of one or more adverse factors. These suture line-in procedures performed only under favorable circumstances, such as in normotensive patients, in patients requiring a short perforation-to-operation interval, in patients with a low volume of peritoneal contaminant fluid, and/or when healthy non-edematous bowel wall was available for repair. For a single perforation, primary closure of the perforation was the procedure of choice. Large or multiple perforations localized in a segment of intestine, perforation in a loop of severely inflamed or friable bowel, and perforations associated with strictures warranted resection of involved intestine and anastomosis. The no suture line procedure (ileostomy), having no risk of suture line leakage, was suitable in adverse circumstances. The ileostomy, however, should not be used indiscriminately because both the procedure itself, as well as the need for another operation to close the ileostomy, inconveniences the patient.

Wound infection was the most frequent complication $(46.8 \%)$. Incidence of wound infection has been variable in various studies, ranging from 19.5 to $95 \%$ [5, 8, 11-15]. The high incidence of wound infection in this series may be attributed to late presentation (median duration of symptoms is six days). Moreover, the hospital caters to a large underprivileged population and provides free treatment. Therefore, wide prevalence of malnutrition and vitamin deficiencies in this stratum of the population may also contribute to the high incidence of wound infection. The current study shows that the wound infection rate can be drastically reduced if the skin incision is not primarily closed at the conclusion of laparotomy. The adoption of this practice, particularly in patients presenting after several days of illness, and if the peritoneal cavity is grossly contaminated by frank pus/feces, is strongly recommended. The wound may be closed on the fourth post-operative day under local anesthesia and sedation.
The study gives insight into contemporary causes of non-traumatic perforation of the small intestine in this part of the world. Typhoid remains the major identifiable cause of small bowel perforation $(46.4 \%)$, the second being tubercular perforation $(12.8 \%)$. In a large proportion of cases $(39.2 \%)$, the underlying cause was not identified and histopathological analysis revealed non-specific inflammation. Similar findings have been reported in a recent review of 12 series of small intestinal perforations [10].

This study highlights the life-saving role of salvage ileostomy for post-operative intestinal leakage in comparison to non-operative treatment. The authors recommend that whenever intestinal leakage is suspected in the postoperative period, urgent exploratory laparotomy must be undertaken and the continuing peritoneal contamination should be controlled by exteriorizing the site of intestinal leak as ileostomy. Good outcome with conservative treatment using octreotide has been reported in the management of post-operative enterocutaneous fistula [14]. However, due to limited resources, conservative treatment involving octreotide and prolonged parenteral nutrition may not be a viable option for majority of our patients.

\section{Conclusion}

Typhoid, nonspecific enteritis, and tuberculosis were three common causes of non-traumatic perforation of the small intestine. Primary repair was the most frequent operative procedure, followed by ileostomy and resection-anastomosis, while wound infection was the most common postoperative complication. Operative procedures undertaken for the management of non-traumatic perforation of the small intestine can be classified into two groups: procedures that leave an intestinal suture line inside the peritoneal cavity and procedures that do not. The no suture line-in procedure seems to be better for patients with adverse factors. Therefore, the operative treatment for small intestinal perforations should be individualized according to preoperative factors and the operative findings. Delayed primary closure of the skin incision helps to reduce the wound infection rate. Salvage ileostomy seems to be a better option for postoperative intestinal leakage.

\section{Acknowledgements}

The authors wish to acknowledge the Department of Pathology, University College of Medical Sciences and Guru Teg Bahadur Hospital, Delhi, for their contributions in performing histopathological examination. 


\section{References}

1. Sharma L, Gupta S, Soin AS, Sikora S, Kapoor V (1991) Generalized peritonitis in India. The Tropical Spectrum. Jpn J Surg 21: 272-277.

2. Ersumo T, W/Meskel Y, Kotisso B (2005) Perforated peptic ulcer in Tikur Anbessa Hospital; a review of 74 cases. Ethiop Med J 43: 9-13.

3. Ayite A, Dosseh DE, Kotakoa G, Tekou HA, James K (2006) Surgical treatment of the single non traumatic perforation of small bowel: excision-suture or resectionanastomosis. Ann Chir 131: 83-84.

4. Uba AF, Chirdan LB, Ituen AM, Mohammed AM (2007) Typhoid intestinal perforation in children: a continuing scourge in a developing country. Pediatr Surg Int. 23: 3339.

5. Shah AA, Wani KA, Wazir BS (1999) The ideal treatment for typhoid enteric perforation: resection anastomosis. Int Surg 84: 35-38.

6. Ara C, Sogutlu G, Yildiz R, Kocak O, Isik B, Yilmaz S, Kirimlioglu V (2005) Spontaneous small bowel perforations due to intestinal tuberculosis should not be repaired by simple closure. J Gastrointestinal Surg 9: 514-517.

7. Pal DK (1998) Evaluation of best surgical procedure in typhoid perforation - an experience of 60 cases. Trop Doc 28: 16-18.

8. Onen A, Dokucu AI, Ciğdem MK, Oztürk H, Otçu S, Yücesan S (2002) Factors affecting morbidity in typhoid intestinal perforation in children. Pediatr Surg Int 18: 696700 .

9. Mechat F and, Zerouali ON (1993) Non-traumatic perforation of small intestine. J Chir 130: 292-296.

10. Gupta S and Kaushik R (2006) Peritonitis - the Eastern experience. World J Emerg Surg. 1: 13.

11. Adesunkanmi AR and, Ajao OG (1997) The prognostic factors in typhoid ileal perforation:a prospective study of 50 patients. J R Coll Surg Edinb 42: 395-399.

12. Mock CN, Amaral J, Visser LE (1992) Improvement in survival from typhoid ileal perforation: Results of 221 operative cases. Ann Surg 215: 244-249.

13. Santillana M (1991) Surgical complications of Typhoid fever: Enteric Perforation. World J Surg. 15: 170-175.

14. Akgun Y, Bac B, Boylu S, Aban N, Tacyildiz I (1995) Typhoid enteric perforation. Br J Surg 82: 1512-1515.

15. Ajao OG (1982) Typhoid perforation: factors affecting mortality and morbidity. Int Surg 67: 317-319.

16. Memon AS, Siddiqui FG (2004) Causes and management of postoperative enterocutaneous fistula. J Coll Physicians Surg Pak. 14: 25-28.

\section{Corresponding author}

Dr. Pankaj Kumar Garg

Department of Surgery

University College of Medical Sciences and Guru Teg Bahadur Hospital

Dilshad Garden, Delhi 110095, India

Phone: +919868399684

Email: dr.pankajgarg@gmail.com

Conflict of interests: No conflict of interests is declared. 